Network Working Group

Request for Comments: 967
M. A. Padlipsky

Mitre Corporation

December 1985

\title{
All Victims Together
}

STATUS OF THIS MEMO

This RFC notes a significant omission from the networking literature and proposes to remedy it. Distribution of this memo is unlimited.

\section{DISCUSSION}

An interesting thing happened the other day. Some people were up visiting from IBM Federal systems Division and, during the course of the conversation, one of them pointed out that they had just as much if not more trouble with the operating system purveyors about making os "changes" in behalf of networking as anyone else. At the time I just observed that it looked as if we were all victims together and went on to the next point, but further reflection prompts me to offer a few thoughts on the topic to the RFC community:

o To us, it's axiomatic that networking code is system code when it has to be.

o To Them, it's anathema.

- We haven't really hit very hard on the point in the literature (although I guess I have made a few strong assertions along those lines, here and there, and it's at least implicit in some of Dave Clark's stuff), unless in my usual slipshod fashion I've just missed seeing it.

- It would probably be responsible of us to rectify the omission (assuming there is one) since the literature is supposed to be the way the researchers educate the practioners.

- Therefore, I propose a new subseries of RFCs on how the networking code was integrated with various OSs, with an eye toward subsequent publication of the collection in the open literature (RFCs being only semi-open, after all). I'll even volunteer to coordinate, at least to the extent of taking offers from people who are willing to tackle various systems and telling them who else is having a bash at the same one for purposes of possible collaboration--and possibly even merging the results of separate efforts if people just send in things they've already done. (I suppose I even have to offer to do a bit of editing, if people want.) 
What I'd like to see emerge is a bunch of little essays along the lines of what I attempted to do on Multics in RFC 928, pp.14-21, which would probably be a waste of electrons to reproduce here, but I will if Jon thinks it's worthwhile at some level. With luck, volunteers will emerge to discuss all of the major operating systems currently on the net and most of the minor ones as well, since one of the most interesting philosophical aspects of the exercise is to see just what cuts and pastes get made to any os if it's networked. My guess is that given more modern systems' tendencies to make adding device drivers more straightforward and to offer interprocess communication primitives at the system level, the likeliest difficulties to encounter would be getting on the process creation path appropriately for Telnet--but that's reasoning ahead of the data. Suffice it to say that each piece should address Host-Host protocol interpreter(s) integration as well as Host-Comm Subnet Processor PI (including device driver, if one), plus something about Telnet and something else about FTP (at least to the extent of whether it's per-user or "monolithic"--on the server side, that is), and, of course, some relevant anatomizing of the os itself.

The moral, it seems to me, is that we have a chance to strike back at the oppressors by showing them what they should be furnishing with their silly off-the-rack systems if they are going to continue to object to our alterations to make the bloody things fit anywhere near right. It's a little extra effort on our part, but it's probably a worthy goal. Indeed, if anybody from IPTO is watching I suppose I'd even go so far as to suggest a pro tem system Integration Task force if I hadn't already volunteered once in this thing and used up my quota.

Think about it.

EDITOR'S NOTE

The editor recalls a session at the 5th Data Communication Symposium (the one at Snowbird) titled "Impact of Networks on Host-System Design and Architecture". (1977) 